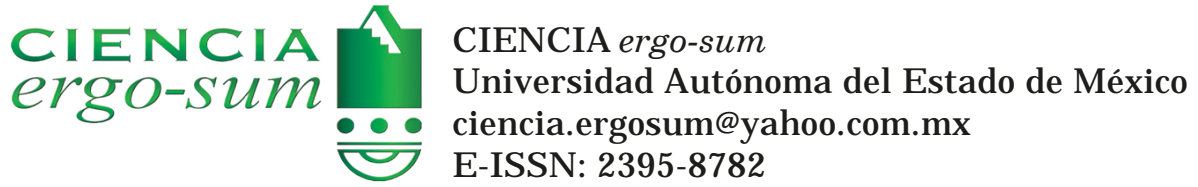

\title{
Tipología de docentes universitarios de acuerdo con su desempeño docente: motivados y no motivados
}

J iménez, Nadia; San-Martín, Sonia

Tipología de docentes universitarios de acuerdo con su desempeño docente: motivados y no motivados

CIENCIA ergo-sum, vol. 26, núm.2, julio-octubre 2019| e50

Universidad Autónoma del Estado de México, México

Esta obra está bajo una Licencia Creative Commons Atribución-NoComercial-SinDerivar 4.0 Internacional.

J iménez, N. y San-Martín S. (2019). Tipol ogía de docentes universitarios de acuerdo con su desempeño docente: motivados y no motivados. CIE N Cl A ergo-sum, 26(2). https://doi .org/10.30878/ces.v26n2a4 


\title{
Tipología de docentes universitarios de acuerdo con su desempeño docente: motivados y no motivados
}

A typology of university teachers according to their job performance: motivated and unmotivated ones

\author{
Nadia Jiménez \\ Universidad de Burgos, España \\ nhjimenez@ubu.es \\ Sonia San-Martin \\ Universidad de Burgos, España \\ sanmargu@ubu.es
}

\section{Resumen}

La labor docente incluye múltiples tareas e implica a diferentes receptores: el docente, la institución educativa y los estudiantes y los compañeros. En este contexto, se obtiene una tipología de profesores universitarios de acuerdo con diferentes aspectos como las condiciones de trabajo percibidas, la evaluación del desempeño docente y la comunicación de la trascendencia y contribución del trabajo. Con este propósito, se obtuvo información de 137 docentes universitarios en España y se realizó un análisis clúster no jerárquico de aglomeración de $k$-medias. Los resultados ofrecen una posible clasificación de los profesores: docentes motivados y docentes desmotivados. Palabras ClaVE: profesor, percepción, sistema de educación universitaria.

\begin{abstract}
The teaching job includes multiple tasks and implies different targets of this work, such as teachers, universities, students and colleagues. The purpose of this research is to obtain a typology of university professors according to several aspects of their job performance: professors' perception of working conditions, evaluation of job performance and their communication about the contribution and significance of their job. With this objective in mind, data were collected from 137 Spanish university professors participating in this study. A non-hierarchical cluster analysis ( $k$-means) was carried out and the results offer a professors' typology of motivated and demotivated professors.
\end{abstract}

KEYWORDS: professor, perception, high-education system.

\section{INTRODUCCIÓN}

Desde la creación del Espacio Europeo de Educación Superior (EEES) se ha pretendido la consecución de diversos cambios en la docencia universitaria. Entre ellos, uno de los más importantes es el cambio en la centralidad del propio proceso educativo (López-Sidro, 2011), que se traslada del docente al alumno. En el marco del EEES, el docente deja de ser un simple transmisor de conocimientos (profesor-instructor) para ser quien enseña a aprender (profesor tutor o guía) (De la Peña y Marcos, 2010) y así tener un papel más transformacional (activo) y menos transaccional (tradicional) en el aula (González-Peiteado y Pino-Juste, 2016). Este cambio de rol exige del docente universitario el desarrollo de habilidades docentes no empleadas hasta ahora (Area, 2009; Torres et al., 2017; Rodrigo-Cano et al., 2017). Por todo ello, el profesor debe buscar la implicación del estudiante para potenciar su comprensión, fomentar su desarrollo personal, su autonomía y mejorar su competencia para aprender a aprender (Ballesta et al., 2011). Valcárcel (2003) destaca que el docente universitario en el EEES debe desarrollar competencias cognitivas, comunicativas, gerenciales, sociales y afectivas. Area (2009) realiza un estudio cualitativo 
mediante grupos de discusión y entrevistas grupales para definir cuatro perfiles de profesores en función de las competencias a desarrollar en el contexto universitario. Concretamente, los dividen en docentes: $a$ ) especialista en docencia, $b$ ) investigador, $c$ ) asociado o especialista en la práctica y $d$ ) multifunción.

En este marco, la actividad docente es una tarea compleja y multidimensional. El profesor debe asumir diferentes roles (docente, investigador, tutor, gestor, evaluador y evaluado) (Oshagbemi, 1997; Area, 2009). Arif e Ilyas (2013) señalan que la percepción de los docentes universitarios sobre su propio trabajo tiene implicaciones más allá de su propia satisfacción laboral. Serey (2006) apunta que para que un trabajador (en este caso un docente) perciba calidad en su vida profesional debe poder apreciar cuatro condiciones: $a$ ) una oportunidad para ejercer talentos y capacidades con independencia e iniciativa propia, $b$ ) una actividad significativa para contribuir, $c$ ) la comprensión de un rol individual para lograr fines comunes y $d$ ) una sensación de orgullo por el trabajo realizado. Rosser (2005) y Arif e Ilyas (2013) destacan que la motivación y la calidad de la vida profesional percibida por los propios docentes diferencia a las instituciones más exitosas de las menos exitosas en el sector universitario.

Así, la percepción de los docentes universitarios sobre su propio trabajo puede ser uno de los aspectos más interesantes que influirán en el hecho de que una universidad destaque frente a otras en el EEES. Escasos son los trabajos que han analizado a los docentes universitarios (Oshagbemi, 1997; Quevedo-Blasco et al., 2014; Arif e Ilyas, 2013; Shah y Sid Nair, 2012) y, menos aún, la posible existencia de tipologías (Oshagbemi, 1997; Rivers et al., 2014). Para Oshagbemi (1997) existen tres tipologías de docentes en el ámbito universitario: felices, satisfechos e infelices. La característica que diferenciaba a los trabajadores infelices de los felices y satisfechos era una mayor preocupación por la promoción y la compensación. Además, destaca que las diferencias entre los grupos no eran causadas por la docencia o la investigación sino por la implicación de tareas como la gestión y la dirección universitarias (Oshagbemi, 1997). En esta misma línea, Timms et al. (2007) señalan que el día a día universitario se ha convertido en un trabajo intensivo debido al incremento de la ratio de estudiantes por docente y a un incremento de tareas de naturaleza administrativa. En este sentido, Yassine-Diab et al. (2014) sugieren que el grado de autonomía profesional influye en la motivación y en el desarrollo personal de los docentes universitarios.

Por otra parte, estudios como el de Rivers et al. (2014) contemplan la labor de los docentes universitarios como un trabajo que permite generar cambios sociales trascendentales, y también atienden al nuevo papel que desempeña el docente al ser un desarrollador de capacidades de aprendizaje e innovación en los estudiantes. Uno de los aspectos que resalta este trabajo es la necesidad de que los docentes encuentren nuevas formas de trabajar. Los autores mencionados proponen la existencia de diversos aspectos a estudiar para poder determinar categorías de docentes universitarios: la estrategia institucional sobre el papel del docente, las características personales, las características profesionales o de empleabilidad, la valoración y aceptación social, el pensamiento crítico, resolutivo y la capacidad para analizar un fenómeno desde diferentes perspectivas. A pesar de que el estudio de Rivers et al. (2014) presenta una clasificación teórica de los docentes, es destacable el señalamiento que hacen a los múltiples aspectos que se deben considerar cuando se estudia el quehacer del profesor universitario.

Para Arife Ilyas (2013), la percepción de la vida laboral de un profesor universitario y su actitud hacia el trabajo pueden ser estudiadas tomando en consideración distintos factores como el valor percibido, el equilibrio entre la vida laboral y la personal y la satisfacción con las relaciones en su vida. Por otra parte, Izquierdo et al. (2011) estudian la actitud de los desempleados en función de factores como el atractivo del trabajo, el autoconcepto personal y profesional y las percepciones sociales como la percepción del empresario. Todos estos factores pueden clasificarse en tres dimensiones: a) las condiciones de trabajo percibidas (por ejemplo disponer de diversas tecnologías y herramientas para el desarrollo de la docencia), b) la evaluación del desempeño docente (por ejemplo la evaluación del docente que realiza la institución) y c) la percepción de trascendencia y contribución a los demás y la sociedad, en general, del trabajo desempeñado (por ejemplo el reconocimiento percibido y autopercepción). En el siguiente apartado, se ofrece una revisión teórica sobre estos tres amplios aspectos tratados en la literatura científica que utilizaremos para abordar el objetivo de obtener una tipología de profesores universitarios de acuerdo con los diferentes aspectos relacionados con el desempeño docente. 


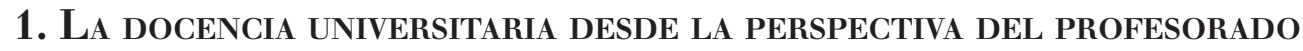

Siguiendo las tres dimensiones que componen el trabajo del docente universitario, trataremos en primer lugar las condiciones en cuanto a herramientas disponibles. El EEES trae consigo una revisión no sólo de los modelos de aprendizaje sino también de las fuentes y recursos empleados (Álvarez, 2009). En este sentido, las tecnologías de la información y de la comunicación (TIC) constituyen herramientas muy potentes para la comunicación entre profesores y alumnos (a través de correo electrónico o redes), para buscar y almacenar información, para fomentar aprendizajes cooperativos o para ayudar a la comprensión de problemas o conceptos complejos (Álvarez, 2009; Fernández y Fernández, 2016). Así, lo pone de manifiesto el estudio empírico de De Pablo y Villaciervos (2005) y Rodrigo-Cano et al. (2017) en el que la integración de las TIC en la enseñanza universitaria es percibida por los profesores como una oportunidad para ofrecer a los estudiantes el control de su propio proceso de aprendizaje y potenciar la interacción entre los docentes y los estudiantes al disponer de más canales de comunicación. Las herramientas tecnológicas son relevantes tanto en la docencia como en el adecuado desarrollo de las tutorías, tanto presenciales como no presenciales, porque permiten intercambiar una gran cantidad de información significativa y se reducen las barreras geográficas, sociales y temporales entre el tutor y el tutorado (Montserrat et al., 2007). La acción tutorial supone el establecimiento de comunicación entre docentes y estudiantes que permite la personalización en el trato dentro del proceso educativo (García-Valcárcel, 2008).

En segundo lugar, otros dos aspectos clave en la docencia son la evaluación de la actividad del profesor y el reconocimiento percibido por los demás respecto a la labor docente realizada. Existe una evaluación de tipo institucional por medio de programas institucionales, la evaluación que hacen los estudiantes de su profesorado y la evaluación de los compañeros y la sociedad a través de la transferencia del conocimiento derivado de actividades como la investigación. Habitualmente, los programas de evaluación del docente, como Docentia o el de mejoramiento del profesorado (por ejemplo PROMEP), incluyen la autoevaluación del profesor, la derivada de las encuestas hechas a estudiantes, la evaluación de los responsables institucionales (como directivos, cuerpos colegiados o gestores académicos) que en múltiples ocasiones son compañeros del docente evaluado (ANECA, 2017; Rueda, 2008). La investigación previa se ha centrado en especial en la segunda evaluación, la realizada por los estudiantes. Así, la literatura ha abordado la evaluación docente por medio de la utilización de valoraciones que el estudiante hace sobre el profesor y sus características (Hamer, 2015). La búsqueda de la calidad y excelencia en las universidades españolas condujo al establecimiento de sistemas de evaluación docente del profesorado y, como consecuencia, a un intenso debate sobre su diseño y efectividad (De Miguel, 1998; Rueda, 2008). Y, en este proceso, la metodología de encuestas a estudiantes ha sido una de las más utilizadas por las instituciones educativas para evaluar a sus docentes (García-Ramos, 1997; Rueda, 2008).

Sin embargo, las encuestas a estudiantes como sistema de evaluación docente no están exentas de críticas (Villa y Morales, 1993), ya sea por el carácter unidireccional de la evaluación, la subjetividad de los alumnos, el uso de cuestionarios de carácter general e indiferenciado o la imposición de la evaluación por parte de la autoridad académica. Como apunta De Miguel (1998), las encuestas a alumnos deben formar parte de un sistema de evaluación de la docencia mucho más amplio y completo que debería de tener en cuenta diversos factores como los contextuales (la institución en la que trabaja el docente), condicionantes iniciales (nivel de carga docente, formación pedagógica, etcétera), el desarrollo y dedicación a los procesos de enseñanza-aprendizaje (relación con alumnos o procesos de innovación que desarrolla) e indicadores relativos a los resultados y experiencia (satisfacción de los estudiantes, colegas, responsables académicos o invitaciones a impartir docencia fuera de la institución). En definitiva, queda clara la relevancia del reconocimiento percibido por el docente del trabajo que realiza, tanto por parte de los estudiantes como por los compañeros o por la institución educativa que lo contrata.

Vila y Kuster (2008) destacan que también es relevante tener en cuenta la experiencia y las técnicas de enseñanza que utilizan los docentes universitarios. Al respecto, señalan que la experiencia en la docencia tiene beneficios importantes como tomar mejores decisiones, explicar mejor la resolución de problemas a través de 
experiencias propias, desarrollar soluciones más rápidamente y mostrar mayor confianza que sus compañeros menos experimentados.

En tercer lugar, si bien las variables detectadas en los estudios comentados sobre evaluación docente como la experiencia, las técnicas de enseñanza o la satisfacción del profesor sirven de guía en nuestra búsqueda de los factores claves para identificar tipologías de docentes, no constituyen el objeto central (Vila y Kuster Boluda, 2008; Serey, 2006). Así, en lo concerniente a esta investigación, Tejedor (2012) señala que el procedimiento de la evaluación del desempeño debe poner un menor énfasis en el control y más en la ayuda de evaluación docente. En este sentido, propone que obtener información basada en las opiniones de otros agentes evaluadores, como son los compañeros, sea esencial. En las universidades, el reconocimiento de los compañeros se produce cada vez más mediante la investigación realizada por el docente (Buela-Casal et al., 2009; Sundaram et al., 2017). Otros trabajos han encontrado que el estudiante valora poco que su docente sea investigador (San Martín et al., 2013); sin embargo, hay organismos como la Agencia Nacional de Evaluación de la Calidad y Acreditación (ANECA) o el Consejo Nacional de Ciencia y Tecnología (Conacyt), formados por compañeros del campo de conocimiento, que se encargan de reconocer la labor del profesor universitario.

Por tanto, se preguntó a los docentes por aspectos en su trabajo universitario tan diversos como el reconocimiento percibido por los alumnos, compañeros y universidad, la utilidad de los diferentes formas de evaluación docente, la utilidad de técnicas docentes en clase, de evaluación continua y de control del aprendizaje, utilidad de herramientas docentes innovadoras y habituales, así como beneficios percibidos de las tutorías y de la experiencia.

El carácter exploratorio de este artículo permite conocer la percepción de los docentes universitarios sobre qué opinan de su labor y cómo consideran que los perciben los receptores de su trabajo (estudiantes, compañeros y universidad). En el siguiente apartado, se detallan las cuestiones relacionadas con el trabajo de campo realizado para este estudio.

\section{Método}

A través de encuestas dirigidas a docentes universitarios pertenecientes a tres universidades españolas (dos públicas y una privada), se obtuvo la información para efectuar el estudio empírico. La información se recogió en junio de 2015. En cuanto al número de encuestas, 137 fueron válidas. Para garantizar el anonimato, las encuestas estuvieron disponibles durante quince días para los participantes en un enlace web remitido por correo electrónico desde cada universidad a todos sus docentes. Los detalles de las características de la muestra se detallan en el cuadro 1 .

\section{1. Instrumento}

A efectos de cumplir con la validez de contenido de las escalas de medida utilizadas para elaborar el cuestionario, éstas se revisaron en la literatura existente y se diseñaron bloques de preguntas referentes a las clases, a las tutorías y a la evaluación (de acuerdo con indicadores de Gargallo et al., 2010 y Casillas y Cabezas, 2010) y a la percepción de reconocimiento o evaluación por parte de los estudiantes e institución (Shah y Sid Nair, 2012; Oshagbemi, 1997). En el caso de las preguntas relacionadas con las herramientas docentes se utilizó como referencia el estudio de Marsh (1987), adaptado al aprendizaje con TIC. Con el fin de medir los beneficios percibidos de la experiencia, la investigación y de elementos característicos del EEES como las guías docentes o la importancia de la evaluación continua, se creó una escala a través de brainstorming entre los miembros del grupo de innovación docente, que cuenta con profesores de distintas universidades. Se utilizaron preguntas cerradas, escalas Likert de cinco posiciones (cuadro 1 y cuadro 2) y preguntas mono-ítem para complementar la información obtenida atendiendo a la depuración y revisión del cuestionario. 


\section{CUADRO 1}

Descripción de la muestra y otras variables de caracterización

\begin{tabular}{|c|c|c|}
\hline Característica & & $\%$ \\
\hline \multirow{10}{*}{ Edad } & De 24 años y menos & 1.5 \\
\hline & De 25 a 29 años & 0.7 \\
\hline & De 30 a 34 años & 7.3 \\
\hline & De 35 a 39 años & 10.9 \\
\hline & De 40 a 44 años & 23.4 \\
\hline & De 45 a 49 años & 24.1 \\
\hline & De 50 a 54 años & 14.6 \\
\hline & De 55 a 59 años & 8.8 \\
\hline & De 60 a 64 años & 7.3 \\
\hline & De 65 años y más & 1.5 \\
\hline \multirow{8}{*}{ Categoría docente } & Titular de universidad & 34.3 \\
\hline & Colaborador/a & 23.4 \\
\hline & Titular de escuela universitaria & 16.8 \\
\hline & Contratado/a doctor & 7.3 \\
\hline & Ayudante doctor & 7.3 \\
\hline & Catedrático/a de universidad & 5.8 \\
\hline & Ayudante & 3.6 \\
\hline & Catedrático/a de escuela universitaria & 1.5 \\
\hline \multirow{10}{*}{ Rama del conocimiento } & Ciencias sociales, políticas, del comportamiento y de la educación & 31.4 \\
\hline & Ingenierías y arquitectura & 21.2 \\
\hline & Ciencias de la naturaleza & 12.4 \\
\hline & Ciencias económicas y empresariales & 10.9 \\
\hline & Química & 7.3 \\
\hline & Biología celular y molecular & 5.1 \\
\hline & Historia, geografía y artes & 4.4 \\
\hline & Matemáticas y física & 3.6 \\
\hline & Ciencias biomédicas & 2.9 \\
\hline & Derecho y jurisprudencia & 0.7 \\
\hline \multirow{5}{*}{ Experiencia docente } & Menos de 5 años & 12.4 \\
\hline & Entre 5 y 10 años & 21.2 \\
\hline & Entre 11 y 20 años & 29.2 \\
\hline & Entre 21 y 30 años & 27.0 \\
\hline & Más de 30 años & 10.2 \\
\hline \multirow{2}{*}{$\begin{array}{l}\text { Opinión sobre la importancia de } \\
\text { la investigación }\end{array}$} & La investigación es muy importante para enriquecer la labor docente & 59.1 \\
\hline & La investigación no enriquece la labor docente & 40.9 \\
\hline \multirow{6}{*}{$\begin{array}{l}\text { Opinión sobre la función de la } \\
\text { guía docente }\end{array}$} & Es una directriz útil para mi docencia & 18.8 \\
\hline & Es un contrato que me vincula con los estudiantes & 36.2 \\
\hline & Es una obligación laboral & 6.5 \\
\hline & Es una herramienta orientadora para los estudiantes & 31.2 \\
\hline & Es una herramienta de coordinación para los docentes & 3.6 \\
\hline & Es una herramienta poco útil para la docencia & 18.1 \\
\hline
\end{tabular}

Fuente: elaboración propia. 


\section{2. Procedimiento}

Con ayuda del paquete estadístico SPSS 22 se realizó el análisis preliminar univariante y bivariante de las variables observables, en el cual no se encuentran anomalías significativas de los datos, por lo que se procede a estudiar la unidimensionalidad de las variables latentes mediante un análisis factorial exploratorio (AFE). En concreto, realizamos un análisis de componentes principales con rotación varimax (cuadro 2). Los resultados permiten confirmar la existencia de diez factores. El factor F1 está representado por la utilidad percibida de las técnicas docentes en clase y el factor $\mathrm{F} 2$ por los beneficios percibidos de las tutorías. La implicación con el uso de herramientas docentes innovadoras está contemplada en el factor F3, la utilidad percibida del programa Docentia en el factor F4, el desarrollo de la evaluación continua en el factor F5, la utilidad percibida de las encuestas a los estudiantes en el factor F6, la utilidad de las técnicas docentes de control del aprendizaje en el factor F7 y la implicación con el uso herramientas docentes habituales en el factor F8. Los beneficios percibidos de la experiencia docente están contemplados en el factor F9 y el reconocimiento percibido en el factor F10.

CUADRO 2

Resultados del AFE (análisis factorial exploratorio)

\begin{tabular}{|c|c|c|c|}
\hline $\begin{array}{l}\text { Factor/ } \\
\% \\
\text { Explicado }\end{array}$ & Cod. & Variables que recoge el factor & Peso \\
\hline \multirow{7}{*}{$\begin{array}{l}\text { F1 } \\
\text { Utilidad percibi- } \\
\text { da de las técnicas } \\
\text { docentes en clase/ } \\
15.5\end{array}$} & V1 & La realización de casos o trabajos en grupos facilita el desarrollo de la clase. & 0.737 \\
\hline & V2 & Las exposiciones orales de trabajos facilitan el desarrollo de la clase. & 0.728 \\
\hline & V3 & La discusión sobre un video visto en clase o en casa facilita el desarrollo de la clase. & 0.721 \\
\hline & V4 & La realización de un proyecto global facilita el desarrollo de la clase. & 0.708 \\
\hline & V5 & En general, el uso de las TIC en las asignaturas facilita el desarrollo de la clase. & 0.504 \\
\hline & V6 & La creación de un blog o foro de la asignatura facilita el desarrollo de la clase. & 0.498 \\
\hline & V7 & La discusión en clase sobre un caso facilitado en papel facilita el desarrollo de la clase. & 0.466 \\
\hline \multirow{5}{*}{$\begin{array}{l}\text { F2 } \\
\text { Beneficios } \\
\text { percibidos de las } \\
\text { tutorías/ } \\
8.9\end{array}$} & V8 & Las tutorías académicas facilitan la accesibilidad del docente al estudiante. & 0.813 \\
\hline & V9 & Las tutorías académicas mejoran el rendimiento de los estudiantes. & 0.798 \\
\hline & V10 & Las tutorías académicas completan y mejoran la formación del estudiante. & 0.698 \\
\hline & V11 & Las tutorías académicas fomentan la asistencia de los estudiantes. & 0.685 \\
\hline & V12 & Las tutorías académicas orientan profesionalmente al estudiante. & 0.580 \\
\hline \multirow{7}{*}{$\begin{array}{l}\text { F3 } \\
\text { Implicación con } \\
\text { el uso de herra- } \\
\text { mientas docentes } \\
\text { innovadoras/ } \\
7.8\end{array}$} & V13 & El uso de la mensajería instantánea está implícito en la actividad docente. & 0.786 \\
\hline & V14 & El uso de la telefonía IP está implícito en la actividad docente. & 0.715 \\
\hline & V15 & $\begin{array}{l}\text { El uso de una plataforma virtual para las asignaturas fuera de la web de la univer- } \\
\text { sidad está implícito en la actividad docente. }\end{array}$ & 0.642 \\
\hline & V16 & El uso de un perfil en redes sociales está implícito en la actividad docente. & 0.608 \\
\hline & V17 & El uso de un blog de la asignatura está implícito en la actividad docente. & 0.589 \\
\hline & V18 & El uso de una pizarra digital está implícito en la actividad docente. & 0.537 \\
\hline & V19 & El uso de una página web propia está implícito en la actividad docente. & 0.484 \\
\hline \multirow{3}{*}{$\begin{array}{l}\text { F4 } \\
\text { Utilidad percibi- } \\
\text { da del programa } \\
\text { Docentia/ } \\
5.9\end{array}$} & V20 & El programa Docentia sirve para estimular la mejora del docente. & 0.859 \\
\hline & V21 & El programa Docentia sirve para evaluar objetivamente al profesorado. & 0.857 \\
\hline & V22 & El programa Docentia sirve para reconocer la labor docente. & 0.848 \\
\hline
\end{tabular}


CUADRO 2 (continúa)

\begin{tabular}{|c|c|c|c|}
\hline \multirow{7}{*}{$\begin{array}{l}\text { F5 } \\
\text { Desarrollo de } \\
\text { la evaluación } \\
\text { continua/ } \\
4.9\end{array}$} & V23 & En la evaluación continua es importante ser muy claro al explicar el sistema. & 0.738 \\
\hline & V24 & Es importante fomentar el aprendizaje a partir de la evaluación continua. & 0.626 \\
\hline & V25 & $\begin{array}{l}\text { En la evaluación continua es importante adaptar el sistema de evaluación a la } \\
\text { asignatura. }\end{array}$ & 0.566 \\
\hline & V26 & $\begin{array}{l}\text { En la evaluación continua es importante impedir el fraude en la realización de } \\
\text { pruebas. }\end{array}$ & 0.544 \\
\hline & V27 & $\begin{array}{l}\text { En la evaluación continua es importante explicar cómo se han corregido las } \\
\text { pruebas. }\end{array}$ & 0.540 \\
\hline & V28 & En la evaluación continua es importante coordinarme con los compañeros de área. & 0.530 \\
\hline & V29 & En la evaluación continua es importante utilizar un sistema múltiple. & 0.490 \\
\hline \multirow{3}{*}{$\begin{array}{l}\text { F6 } \\
\text { Utilidad percibida } \\
\text { de las encuestas } \\
\text { a los estudiantes/ } \\
4.3\end{array}$} & V30 & $\begin{array}{l}\text { Las encuestas que se realizan a los estudiantes son útiles para evaluar objetiva- } \\
\text { mente al profesorado. }\end{array}$ & 0.866 \\
\hline & V31 & $\begin{array}{l}\text { Las encuestas que se realizan a los estudiantes son útiles para reconocer la labor } \\
\text { docente del profesorado. }\end{array}$ & 0.813 \\
\hline & V32 & $\begin{array}{l}\text { Las encuestas que se realizan a los estudiantes son útiles para estimular la mejora } \\
\text { docente del profesorado. }\end{array}$ & 0.804 \\
\hline \multirow{6}{*}{$\begin{array}{l}\text { F7 } \\
\text { Utilidad de las } \\
\text { técnicas docentes } \\
\text { de control del } \\
\text { aprendizaje/ } \\
4.1\end{array}$} & V33 & La realización de un examen presencial facilita el desarrollo de la asignatura. & 0.722 \\
\hline & V34 & La realización de ejercicios en papel facilita el desarrollo de la asignatura. & 0.688 \\
\hline & V35 & Las clases teóricas facilitan el desarrollo de la asignatura. & 0.645 \\
\hline & V36 & $\begin{array}{l}\text { La realización de controles parciales sin puntuación facilita el desarrollo de la } \\
\text { asignatura. }\end{array}$ & 0.617 \\
\hline & V37 & La realización de exámenes virtuales facilita el desarrollo de la asignatura. & 0.513 \\
\hline & V38 & $\begin{array}{l}\text { La realización de ejercicios con un software especializado facilita el desarrollo } \\
\text { de la asignatura. }\end{array}$ & 0.486 \\
\hline \multirow{3}{*}{$\begin{array}{l}\text { F8 } \\
\text { Implicación } \\
\text { con el uso herra- } \\
\text { mientas docentes } \\
\text { habituales/ } \\
3.3\end{array}$} & V39 & El uso del cañón o proyector está implícito en la actividad docente. & 0.707 \\
\hline & V40 & El uso del correo electrónico está implícito en la actividad docente. & 0.692 \\
\hline & V41 & El uso del internet está implícito en la actividad docente. & 0.599 \\
\hline \multirow{3}{*}{$\begin{array}{l}\text { F9 } \\
\text { Beneficios perci- } \\
\text { bidos de la expe- } \\
\text { riencia docente/ } \\
3.2\end{array}$} & V42 & La experiencia docente fomenta la formación especializada del profesorado. & 0.560 \\
\hline & V43 & La experiencia docente mejora el trato a los estudiantes por el profesorado. & 0.441 \\
\hline & V44 & La experiencia docente mejora las habilidades pedagógicas del profesorado. & 0.359 \\
\hline \multirow{3}{*}{$\begin{array}{l}\text { F10 } \\
\text { Reconocimiento } \\
\text { percibido/ } \\
2.9\end{array}$} & V45 & Los compañeros reconocen mi labor docente. & 0.716 \\
\hline & V46 & La universidad reconoce mi labor docente. & 0.520 \\
\hline & V47 & Los estudiantes reconocen mi labor docente. & 0.400 \\
\hline
\end{tabular}

Fuente: elaboración propia.

Para descartar problemas de sesgo común se utilizó el método de un factor de Harman. Siguiendo las recomendaciones de Podsakoff et al. (2003), mediante un AFE de componentes principales, el primero explicaba únicamente $16 \%$ y descarta la posible existencia de sesgo del método común. Posteriormente, se realizó un análisis factorial confirmatorio (AFC) por ser el método más adecuado para validar el modelo de medida (Podsakoff et al., 2003). 
El análisis factorial confirmatorio realizado con el programa LISREL 8.7 trata de apreciar la validez del modelo de medida en el que se especifican las relaciones directas entre las variables observables y los constructos subyacentes (Jöreskog y Sörbom, 1993). Los resultados obtenidos sirven para obtener las escalas definitivas y determinar la validez convergente y discriminante del modelo de medida (Jöreskog y Sörbom, 1993). La validez de las variables observables para medir cada constructo se realiza en función de los coeficientes lambda $(\lambda)$, el estadístico $t$ y el coeficiente $R^{2}$. Se puede corroborar que la relación directa entre cada factor y sus ítems correspondientes son significativas a 95\% en todos los casos $(t>1.96)$. La reespecificación del modelo se fundamenta en las siguientes medidas de bondad de ajuste: estadístico chi cuadrado $\left(\chi^{2}\right)$, error de aproximación cuadrático medio (RMSEA, Root Mean Square Error of Approximation), índice de ajuste normado (NFI, Normed Fit Index), índice de ajuste comparativo (CFI, Comparative Fit Index), índice de ajuste incremental (IFI, Incremental Fit Index) e índice de bondad del ajuste (GFI, Goodness of Fit Index).

Los resultados del modelo ajustado se muestran en el cuadro 3, donde también se informa de los valores del coeficiente alfa de Cronbach, la fiabilidad compuesta y la varianza extraída correspondientes a cada constructo. Para todas las escalas, los valores que arrojan esos tres indicadores superan los valores aceptables de 0.7, 0.6 y 0.5 respectivamente (Bagozzi y Yi, 1988). En cuanto a la validez discriminante, se corroboró que el valor de la varianza extraída fuese siempre mayor que las correlaciones al cuadrado con otros constructos (Bagozzi y Yi, 1988). Finalmente, con las escalas depuradas de cada variable obtuvimos una puntuación factorial que resume la información de todos los ítems que componen cada uno de los diez factores. Una vez que hemos obtenido un modelo de medida satisfactorio y las puntuaciones factoriales, el siguiente paso consiste en obtener la tipología de docentes a través de un análisis cluster.

CUADRO 3

Resultados del AFC ajustado*

\begin{tabular}{|c|c|c|c|c|c|c|c|c|}
\hline Variable latente & $\begin{array}{l}\text { Variable } \\
\text { observable }\end{array}$ & $\lambda$ & $\begin{array}{l}\text { Error de } \\
\text { medida }\end{array}$ & $t$ & $R^{2}$ & $\alpha$ & $\begin{array}{l}\text { Fiabilidad } \\
\text { compuesta }\end{array}$ & $\begin{array}{l}\text { Varianza } \\
\text { extraída }\end{array}$ \\
\hline \multirow{7}{*}{$\begin{array}{l}\text { Utilidad percibida de } \\
\text { las técnicas docentes } \\
\text { en clase }\end{array}$} & V1 & 1.000 & 0.113 & - & 0.701 & \multirow{7}{*}{0.787} & \multirow{7}{*}{0.914} & \multirow{7}{*}{0.650} \\
\hline & $\mathrm{V} 2$ & 0.868 & 0.196 & 11.48 & 0.608 & & & \\
\hline & V3 & 0.779 & 0.491 & 9.91 & 0.509 & & & \\
\hline & V4 & 0.772 & 0.418 & 9.80 & 0.502 & & & \\
\hline & V5 & 0.609 & 0.468 & 7.29 & 0.332 & & & \\
\hline & V6 & \multicolumn{4}{|c|}{ Eliminado } & & & \\
\hline & V7 & 0.505 & 0.246 & 5.87 & 0.436 & & & \\
\hline \multirow{5}{*}{$\begin{array}{l}\text { Beneficios percibidos } \\
\text { de las tutorías }\end{array}$} & V8 & 0.467 & 0.174 & 10.72 & 0.543 & \multirow{5}{*}{0.812} & \multirow{5}{*}{0.892} & \multirow{5}{*}{0.638} \\
\hline & V9 & 0.817 & 0.241 & 15.81 & 0.814 & & & \\
\hline & V10 & 0.962 & 0.487 & 7.76 & 0.354 & & & \\
\hline & V11 & 1.000 & 0.457 & - & 0.688 & & & \\
\hline & V12 & 0.635 & 0.466 & 10.21 & 0.513 & & & \\
\hline \multirow{7}{*}{$\begin{array}{l}\text { Implicación con el } \\
\text { uso de herramientas } \\
\text { docentes innovadoras }\end{array}$} & V13 & 1.000 & 0.324 & - & 0.759 & \multirow{7}{*}{0.745} & \multirow{7}{*}{0.877} & \multirow{7}{*}{0.603} \\
\hline & V14 & 0.905 & 0.336 & 12.20 & 0.676 & & & \\
\hline & V15 & 0.617 & 0.365 & 6.06 & 0.400 & & & \\
\hline & V16 & 0.554 & 0.417 & 7.44 & 0.397 & & & \\
\hline & V17 & \multicolumn{4}{|c|}{ Eliminado } & & & \\
\hline & V18 & 0.537 & 0.396 & 6.55 & 0.582 & & & \\
\hline & V19 & \multicolumn{4}{|c|}{ Eliminado } & & & \\
\hline
\end{tabular}




\begin{tabular}{|c|c|c|c|c|c|c|c|c|}
\hline & & & UADRC & (conti & & & & \\
\hline & V20 & 1.000 & 0.208 & - & 0.792 & & & \\
\hline Utilidad percibida del & V21 & 0.985 & 0.081 & 21.72 & 0.919 & 0.923 & 0.971 & 0.917 \\
\hline & V22 & 0.915 & 0.174 & 18.46 & 0.826 & & & \\
\hline & V23 & 1.000 & 0.134 & - & 0.660 & & & \\
\hline & V24 & 0.881 & 0.311 & 9.63 & 0.479 & & & \\
\hline & V25 & 0.772 & 0.471 & 8.85 & 0.429 & & & \\
\hline $\begin{array}{l}\text { Desarrollo de la eva- } \\
\text { luación continua }\end{array}$ & V26 & & Elim & do & & 0.762 & 0.919 & 0.607 \\
\hline & $\mathrm{V} 27$ & 0.821 & 0.473 & 10.41 & 0.527 & & & \\
\hline & V28 & 0.635 & 0.356 & 11.44 & 0.589 & & & \\
\hline & V29 & 0.722 & 0.321 & 7.58 & 0.400 & & & \\
\hline Utilidad percibida de & V30 & 1.000 & 0.113 & - & 0.887 & & & \\
\hline las encuestas a los & V31 & 0.941 & 0.196 & 17.38 & 0.804 & 0.906 & 0.940 & 0.839 \\
\hline estudiantes & V32 & 0.865 & 0.311 & 14.50 & 0.689 & & & \\
\hline & V33 & 1.000 & 0.308 & - & 0.692 & & & \\
\hline & V34 & 0.741 & 0.529 & 8.963 & 0.417 & & & \\
\hline Utilidad de las técni- & V35 & 0.866 & 0.388 & 10.82 & 0.612 & & & \\
\hline $\begin{array}{l}\text { cas docentes de con- } \\
\text { trol del aprendizaje }\end{array}$ & V36 & 0.501 & 0.486 & 5.685 & 0.333 & 0.705 & 0.850 & 0.598 \\
\hline & V37 & & Elim & & & & & \\
\hline & V38 & & Elim & & & & & \\
\hline & V39 & 1.000 & 0.376 & - & 0.624 & & & \\
\hline Implicación con el uso & V40 & 0.955 & 0.241 & 9.87 & 0.514 & 0.704 & 0.925 & 0.806 \\
\hline & V41 & 0.877 & 0.377 & 12.20 & 0.664 & & & \\
\hline Beneficios & V42 & 1.000 & 0.364 & - & 0.710 & & & \\
\hline percibidos de la expe- & V43 & 0.673 & 0.409 & 13.37 & 0.733 & 0.738 & 0.870 & 0.696 \\
\hline riencia docente & V44 & 0.901 & 0.217 & 8.12 & 0.398 & & & \\
\hline & V45 & 1.000 & 0.392 & - & 0.608 & & & \\
\hline $\begin{array}{l}\text { Reconocimiento per- } \\
\text { cibido }\end{array}$ & V46 & 0.877 & 0.195 & 13.12 & 0.805 & 0.700 & 0.943 & 0.848 \\
\hline & V47 & 0.695 & 0.208 & 10.72 & 0.504 & & & \\
\hline Bondad del ajuste & & 93.94 & 0.00 ); & $\begin{array}{l}\text { MSEA }= \\
\text { FI }=0\end{array}$ & $\begin{array}{l}4 ; \mathrm{NFI} \\
\mathrm{GFI}=\end{array}$ & $90 ; \mathrm{CFI}$ & 91 ; IF & .90 \\
\hline
\end{tabular}

Fuente: elaboración propia.

Nota: ${ }^{*} \mathrm{El} R^{2}$ de seis variables eran bajos por lo que se procedió a la eliminación de estos ítems.

\section{Resultados}

\section{1. Tipologías de docentes universitarios}

En este apartado se lleva a cabo un análisis cluster con el fin de agrupar a los profesores encuestados con base en los factores obtenidos en el apartado anterior. El análisis cluster es una clasificación o agrupamiento de una población de $n$ individuos en $g$ grupos según determinadas características. Por tanto, para crear tipologías de docentes recurrimos al análisis cluster no jerárquico de aglomeración K-means. El análisis cluster es un método de partición iterativa que permite probar sucesivamente divisiones de la muestra. La solución definitiva elegida para este estudio es la de dos conglomerados que atiende al tamaño de los grupos, a la posibilidad de interpretación de los resultados, al grado 
de significación de cada factor en cada uno de los análisis (ANOVA) y a la posición de centroides finales (cuadro 4). El grado de significación de las variables y factores (análisis ANOVA) corrobora que todos son suficientemente representativos a un nivel de confianza de 95\%. Los valores del estadístico $F$ indican que las mayores variaciones entre los grupos vienen producidas especialmente por el reconocimiento percibido, los beneficios percibidos de las tutorías, los beneficios percibidos de la experiencia y el desarrollo de la evaluación continua (cuadro 4).

CUADRO 4

Análisis ANOVA y centros de los conglomerados finales

\begin{tabular}{lcccc}
\hline Variable latente & Estadístico F & $\begin{array}{c}\text { Sig. } \\
(95 \%)\end{array}$ & \multicolumn{2}{c}{ Centros finales } \\
\cline { 5 - 5 } & & 0.000 & 0.25696 & -0.42004 \\
\hline $\begin{array}{l}\text { Utilidad percibida de las técnicas docentes en } \\
\text { clase }\end{array}$ & 16.47 & 0.000 & 0.39248 & -0.64156 \\
$\begin{array}{l}\text { Beneficios percibidos de las tutorías } \\
\text { Implicación con el uso de herramientas docentes }\end{array}$ & 10.88 & 0.001 & 0.21249 & -0.34734 \\
innovadoras & 18.66 & 0.000 & 0.27155 & -0.44388 \\
$\begin{array}{l}\text { Utilidad percibida del programa Docentia } \\
\text { Desarrollo de la evaluación continua }\end{array}$ & 36.23 & 0.000 & 0.35846 & -0.58594 \\
$\begin{array}{l}\text { Utilidad percibida de las encuestas a los estu- } \\
\text { diantes }\end{array}$ & 12.83 & 0.000 & 0.22962 & -0.37533 \\
$\begin{array}{l}\text { Utilidad de las técnicas docentes de control del } \\
\text { aprendizaje }\end{array}$ & 17.68 & 0.000 & 0.26518 & -0.43347 \\
$\begin{array}{l}\text { Implicación con el uso herramientas docentes } \\
\text { habituales }\end{array}$ & 17.55 & 0.000 & 0.26429 & -0.43201 \\
$\begin{array}{l}\text { Beneficios percibidos de la experiencia docente } \\
\text { Reconocimiento percibido }\end{array}$ & 26.51 & 0.000 & 0.31571 & -0.51607 \\
\hline
\end{tabular}

Fuente: elaboración propia.

El primer grupo conformado por 62\% de la muestra es el de los docentes motivados, quienes tienen puntuaciones positivas en todos los factores analizados. Un segundo grupo, el de los docentes desmotivados, está conformado por 38\% restante de la muestra, que refleja puntuaciones negativas en todos los factores analizados. En seguida presentamos el perfil más característico de cada uno de los dos grupos en función de sus características y las percepciones de cada una de las actividades que desarrollan los docentes universitarios al asumir múltiples roles en el EEES (como docente, investigador, tutor, gestor, evaluador y evaluado) con ayuda de un análisis descriptivo de frecuencias realizado.

a) Grupo 1. Docentes motivados: atendiendo los valores positivos de los centroides finales, este primer grupo considera útiles las técnicas docentes en clase y de control del aprendizaje; asimismo, otorga importancia a la evaluación continua y a las tutorías. En sus clases está implícito el uso de herramientas docentes innovadoras y habituales, les parece esencial que se reconozca su labor y el ser evaluados mediante el programa Docentia o las encuestas a los estudiantes. Además, perciben importantes beneficios de su experiencia docente.

Es destacable que $70.6 \%$ de los docentes motivados opina que es muy importante la investigación para enriquecer la labor docente. Los docentes de este grupo señalan que la mejora continua se consigue a través de cursos de especialización (50.6\%). Respecto al uso de las nuevas tecnologías, $71.1 \%$ de los docentes motivados utiliza las TIC todos los días. Las herramientas que más utilizan son correo electrónico (72.9\%) y la plataforma virtual o sitio web de su universidad (27.1\%). Para un 30\%, la guía docente es una herramienta orientadora para los estudiantes. 
Respecto al perfil característico que tienen los docentes de este grupo, son titulares de universidad (32.9\%) y asociados (27.1\%), con una edad entre 40 y 54 años (58.8\%) y 54.1\% pertenece a áreas del conocimiento de ciencias sociales y lleva de 5 a 20 años impartiendo docencia (63.5\%).

b) Grupo 2. Docentes desmotivados: atendiendo los valores negativos de los centroides finales, este segundo grupo de docentes considera menos útiles las técnicas docentes utilizadas para facilitar el desarrollo de la clase o el control del aprendizaje y percibe menor utilidad en la evaluación continua y en las tutorías. En sus clases no destaca el uso de herramientas docentes y no parece valorar el reconocimiento de su labor mediante el programa Docentia o las encuestas a los estudiantes. Además, minimiza los beneficios aportados por la experiencia docente.

De los docentes desmotivados, 59.7\% considera poco importante la investigación para enriquecer la labor docente. Quienes integran este grupo señalan que la mejora continua del docente se consigue a través de cursos pedagógicos e incentivos laborales (51.9\%). Respecto al uso de las nuevas tecnologías, 35\% de los docentes desmotivados utiliza las TIC alguna vez a la semana y $28.8 \%$ regularmente, pero no todos los días. Para un $40.4 \%$, la guía docente es un contrato con los estudiantes.

Respecto al perfil característico que tienen los docentes de este grupo, son principalmente titulares de universidad (36.5\%) y doctores contratados (21.2\%), con una edad predominante entre 45 y 59 años (69.2\%), 34.6\% pertenece a áreas del conocimiento de ingeniería y $32.7 \%$ a ciencias económicas y empresariales. Este grupo de docentes lleva entre 11 y 30 años impartiendo docencia (58.5\%).

\section{Conclusiones}

Tal y como se ha planteado al inicio, el objetivo era obtener una tipología de docentes atendiendo diferentes aspectos implícitos en el proceso de enseñanza-aprendizaje dentro del marco del EEES y a los múltiples roles que desempeñan los docentes en su trabajo universitario. En la literatura hay pocos trabajos centrados en la percepción del docente sobre su propio trabajo para obtener una tipología de profesores (Oshagbemi, 1997; Rivers et al., 2014; Area, 2009).

Nuestros resultados muestran que se pueden identificar dos tipos de docentes. Hay un primer grupo más motivado con su trabajo docente y un segundo grupo más desmotivado. Además de la clara diferenciación que producen las valoraciones positivas o negativas en los diez factores analizados para poder clasificar a los docentes, es interesante la diferencia en el perfil de un docente más motivado y menos motivado en el ámbito universitario. En este contexto, el docente más motivado es aquel que busca ser accesible a sus estudiantes y a la vez implicar en el proceso de enseñanza-aprendizaje el uso de todas TIC a su alcance. En este grupo de docentes que hemos denominado motivados, respecto del grupo de desmotivados, hay más profesores jóvenes, asociados y que tienen menos años de experiencia. En el grupo de docentes menos motivados, hay más profesores con más años impartiendo docencia, funcionarios o con un contrato indefinido como profesor universitario. Su implicación con el uso de nuevas tecnologías o técnicas docentes innovadoras en sus clases es menor. Este resultado contradice la aportación del estudio de Vila y Kuster Boluda (2008), que sugiere una relación positiva entre la experiencia y el uso de las TIC, pero no distingue los profesores por su grado de motivación.

Dichos resultados están en consonancia con estudios previos como los de Oshagbemi (1997), Arif e Ilyas (2013) y Yassine-Diab et al. (2014), en los que una de las principales diferencias entre los docentes motivados y desmotivados es la percepción por los profesores desmotivados de su propio trabajo como vinculado a imposiciones institucionales y sociales o la percepción de la trascendencia de cumplir con múltiples roles para contribuir al desarrollo social desde el proceso de enseñanza-aprendizaje de los individuos. En este sentido, es ilustrativo que mientras los docentes motivados entienden la guía docente como una herramienta orientativa para los estudiantes, que no coarta su autonomía para innovar en el proceso de enseñanza-aprendizaje, para los docentes desmotivados es un contrato con los estudiantes que posiblemente los límiten al cumplimiento de un programa curricular que debería ser actualizado o mejorado desde su perspectiva, lo anterior al haber desarrollado un espíritu más crítico como consecuencia de su experiencia en la docencia universitaria. 
Una recomendación práctica que deriva de este artículo es la necesidad de las instituciones universitarias en diferenciar las medidas de reconocimiento del desempeño según el tipo de profesor. Así, parece conveniente estimular a los docentes desmotivados mediante cursos pedagógicos e incentivos laborales y proporcionar a los docentes más motivados reconocimiento y cursos de especialización. En ese aspecto, puede influir en la diferenciación entre los grupos la edad de los docentes y la antigüedad en las instituciones universitarias.

Entre las limitaciones, debemos señalar el reducido tamaño muestral, el hecho de que sólo se han considerado tres universidades para obtener información, así como la baja participación de los docentes para responder a la encuesta que impide la generalización de los resultados. Futuros estudios podrían tratar de diseñar un modelo causal que relacione las variables de este artículo para explicar la motivación docente y obtener resultados más concluyentes sobre cómo mejorar la propia percepción de su trabajo en los docentes universitarios en el marco del EEES. Como línea futura, se podría complementar esta información obtenida directamente del docente con información proporcionada por los estudiantes y por la propia institución, así como comparar los resultados con otros países. Otra vía de ampliación y mejora radica en el análisis de las posibles diferencias entre docentes motivados y desmotivados de universidades públicas contra universidades privadas, de enseñanza presencial contra enseñanza online, por facultades o áreas de conocimiento o por figuras contractuales (por ejemplo la de asociado, ayudante doctor, contratado o funcionario). Finalmente, sería interesante analizar si hay relación entre la motivación de los docentes y la evaluación que reciben por parte de los alumnos. Con esto, se conocería si los estudiantes detectan esa motivación y lo trasladan después a su evaluación.

\section{Análisis Prospectivo}

El trabajo y los resultados obtenidos desde la perspectiva del docente universitario suponen un reto para quienes gestionan la enseñanza de individuos en el nivel superior; de este modo, deben organizar a sus recursos humanos de la forma más eficiente posible buscando lograr su satisfacción, adecuada actitud en el trabajo y transferencia de su motivación a estudiantes, compañeros y la sociedad en general. Pocos artículos se han centrado en el punto de vista del docente; por esto, su autorreflexión es necesaria para lograr una mejora en su rendimiento y motivación. Aunque los cambios en la enseñanza universitaria sugieren un empoderamiento del estudiante en su rol central en el proceso enseñanza-aprendizaje, un docente motivado sigue siendo el pilar clave facilitador del aprendizaje del estudiante y orientador en su vida profesional. La institución tiene como deber conocer y valorar la motivación y actitud del docente para ayudarlo en su trabajo y corregir, si es necesario, las deficiencias reales o percibidas en ese desempeño. Este análisis incidirá en todo tipo de enseñanza, pero de manera decisiva en el contacto presencial del docente con el estudiante y en las relaciones laborales entre todos los partícipes que forman la comunidad universitaria y cuyo objetivo final es ofrecer una formación excelente y autocrítica en todos los niveles educativos.

\section{Agradecimientos}

Los autores agradecen el apoyo del proyecto con referencia ECO2017-82107-R.

\section{ReFerencias}

Álvarez, V. (2009). Perfiles y competencias docentes requeridos en el contexto actual de la educación universitaria. Revista Española de Orientación y Psicopedagogía, 20(3), 270-283.

ANECA (Agencia Española de Evaluación de la Calidad y Acreditación) (2017). Programas de evaluación Docentia. Disponible en http://www.aneca.es/Programas-de-evaluacion/DOCENTIA. Consultado en diciembre de 2017. 
Area, E. (2009). Perfiles docentes para el espacio europeo de educación superior (EEES) en el ámbito universitario español. Revista electrónica de investigación y evaluación educativa, 15(1), 1-18.

Arif, S. \& Ilyas, M. (2013). Quality of work-life model for teachers of private universities in Pakistan. Quality Assurance in Education, 21(3), 282-298.

Bagozzi, R. P. \& Yi, Y. (1988). On the evaluation of structural equation models. Journal of the Academy of Marketing Science, 16(1), 74-94.

Ballesta, F. J., Izquierdo, T. y Romero, B. E. (2011). Percepción del alumnado de pedagogía ante el uso de metodologías activas. Educatio Siglo XXI, 29(2), 353-368.

Buela-Casal, G., Paz Bermúdez, M. D. L., Sierra, J. C., Quevedo-Blasco, R. y Castro, Á. (2009). Ranking de 2008 en productividad en investigación de las universidades públicas españolas. Psicothema, 21(2), 304-312.

Casillas, S. y Cabezas, M. (2010). Distintas concepciones sobre la "buena docencia universitaria”. Revista de ciencias de la educación, 223, 351-369.

De la Peña, M. M. y Marcos, M. (2010). La clase magistral como metodología docente aplicable al EEES, en E. M. Rubio, M. M. Pardo y M. Farias (Ed.). Buenas prácticas para la docencia del Derecho adaptada al ECTS. Murcia: Ediciones de la Universidad de Murcia.

De Miguel, M. (1998). La evaluación del profesorado universitario. Criterios y propuestas para mejorar la función docente. Revista de Educación, 315, 67-83.

De Pablo, J. y Villaciervos, P. (2005). El EEES y las tecnologías de la información y la comunicación. Percepciones y demandas del profesorado. Revista de Educación, 337, 99-124.

Fernández, F. J. y Fernández, M. J. (2016). Los docentes de la Generación Z y sus competencias digitales. Comunicar, 46, 97-105.

García-Ramos, J. M. (1997). Valoración de la competencia docente del profesor universitario. Una aproximación empírica. Revista Complutense de Educación, 8(2), 82-108.

García-Valcárcel, A. (2008). La tutoría en la enseñanza universitaria y la contribución de las TIC para su mejora. Relieve, 14(2), 1-14.

Gargallo, B., Sánchez, F., Ros, C. y Ferreras, A. (2010). Estilos docentes de los profesores universitarios. La percepción de los alumnos de los buenos profesores. Revista Iberoamericana de Educación, 51(4), 1-16.

González-Peiteado, M. y Pino-Juste, M. (2016). Los estilos de enseñanza: construyendo puentes para transitar las diferencias individuales del alumnado. Revista Complutense de Educación, 27(3), 1175-1191.

Hamer, F. A. (2015). La percepción del buen profesor en alumnos de nuevo ingreso a la enseñanza universitaria: el caso de ETEA (Córdoba). Revista Complutense de Educación, 26(2), 227-240.

Izquierdo, T., Hernández, F., Maquillón, J. J. y López, O. (2011). Adaptación y validación de la escala de actitudes hacia el trabajo en desempleados mayores de 45 años. Revista iberoamericana de diagnóstico y evaluación psicológica, 2(32), 105-122.

Jöreskog, K. G. y Sörbom, D. (1993). LISREL 8: Structural equation modeling with the SIMPLIS command language. Chicago: Scientific Softivare International

López-Sidro, A. (2011). Reflexiones sobre los desafíos de Bolonia y la misión del profesor universitario. Entre la innovación y la tradición. Revista Jurídica de Investigación e Innovación Educativa, 4, 23-44.

Marsh, H. W. (1987). Students' evaluations of university teaching: Research findings, methodological issues, and directions for future research. International Journal of Education Research, 11, 253-388.

Montserrat, S., Gisbert Cervera, M. e Isus, S. (2007). E-tutoría: uso de las tecnologías de la información y comunicación para la tutoría académica universitaria. Teoría de la educación: educación y cultura en la sociedad de la información, 8(2), 31-54.

Oshagbemi, T. (1997). Job satisfaction profiles of university teachers. Journal of Managerial Psychology, 12(1), 27-39. 
Podsakoff, P. M., MacKenzie, S. B., Lee J. Y. y Podsakoff, N. P. (2003). Common method biases in behavioral research: A critical review of the literature and recommended remedies. Journal of Applied Psychology, 88(5), 879-903.

Quevedo-Blasco, R., Buela-Casal, G. y Ariza, T. (2014). Evaluación de la satisfacción del profesorado de ciencias con la adaptación al espacio europeo de educación superior. Educación XX1,18(1).

Rivers, A. B., Nie, M., \& Armellini, A. (2014). University teachers' conceptions of "Changemaker": A starting point for embedding social innovation in learning and teaching. Education+Training, 57(5), 588-600.

Rodrigo-Cano, D., Iglesias-Onofrio, M. y Aguaded, I. (2017). Metodologías participativas en la nube: la "g-Google" vs. la "Generación X" en la Web 2.0. Revista Complutense de Educación, 28(1), 223-237.

Rosser, V.J. (2005). Measuring the change in faculty perceptions over time: an examination of their worklife and satisfaction. Research in Higher Education, 46(1), 81-107.

Rueda, M. (2008). La evaluación del desempeño docente en la universidad. Revista Electrónica de Investigación Educativa, 10(3), 1-15.

San Martín, S., Santamaría, M., Ibáñez, J., Hoyuelos, F. J., Jerónimo, E. (2013). Variables definitorias del perfil del profesor universitario ideal desde la perspectiva de los estudiantes pre-universitarios. Educación XXI, $17-2,193-215$.

Serey, T. T. (2006). Choosing a robust quality of work life. Business Forum, 27(2), 7-10.

Shah, M., y Sid Nair, C. (2012). The changing nature of teaching and unit evaluations in Australian universities. Quality Assurance in Education, 20(3), 274-288.

Sundaram, P. A. y González, M. E. B. (2017). Investigación Académica: la ruta para el éxito docente y la clave para la enseñanza. Gestión de la Educación, 7(1), 179-189.

Tejedor, F.J. T. (2012). Evaluación del desempeño docente. Revista Iberoamericana de Evaluación Educativa, 5(1), 318-327.

Timms, C., Graham, D., \& Cottrell, D. (2007). I just want to teach. Queensland independent school teachers and their workload. Journal of Educational Administration, 45(5), 569-586.

Torres, C. I., Alcántar, M. D. R. C., Freixes, N. G. y Franco, B. M. G. (2017). Análisis del saber de los estudiantes universitarios sobre su proceso de aprendizaje. CIENCIA ergo-sum, 24(3), 207-217.

Valcárcel, M. (2003). La preparación del profesorado universitario español para la convergencia europea en educación superior. Educatio, 23, 209-213.

Vila, N. y Kuster Boluda, I. (2008). Cross cultural marketing teachers' profile. Multicultural Education \& Technology Journal, 2(3), 140-155.

Villa, A. y Morales, P. (1993). La evaluación del profesor. Una visión de los principales problemas y enfoques en diversos contextos. Vitoria: Servicio de Publicaciones del Gobierno Vasco.

Yassine-Diab, N., Monnier, N. y Lavinal, F. (2014). Pilot study on university English teachers' professional autonomy in France. International Journal of Organizational Analysis, 22(2), 110-125.

\section{BY-NC-ND}

